\title{
Consignes en scène
}

Maud Verdier ${ }^{1}$

Laboratoire Praxiling, Université Paul Valéry Montpellier 3

\begin{abstract}
Résumé. En privilégiant le travail de plateau, les arts vivants contemporains ont profondément transformé le statut des répétitions au théâtre. Celles-ci supposent une collaboration intense entre metteurs en scène et comédiens dans le temps et l'espace de la performance. Cherchant à saisir la

« machinerie » théâtrale (Kantor, [1977] 2004), on s'intéresse aux consignes des metteurs en scène et à leur réception par les comédiens. L'article propose une analyse séquentielle et multimodale d'une répétition, recueillie dans le cadre d'une enquête ethnographique menée au sein d'une troupe de théâtre incluant l'observation et l'enregistrement audiovisuel du travail scénique.
\end{abstract}

\begin{abstract}
The research program dedicated to the performance studies emphasized the need to investigate the dimension of performance at the heart of the theatre. In the contemporary performing arts, rehearsals gain further importance due to the centrality of stage work. On the basis of a video- recorded corpus of the French theatre company's rehearsals, this article explores the collaborative nature of rehearsal work and the multimodal resources with which the stage director carries out his work during one particular rehearsal sequence. The data and the results contribute to our understanding of the nature of the collaborative and multimodal rehearsal work in providing performers with adequate patterns to perform the dramatic action in real time and space.
\end{abstract}

\section{Introduction}

Dans le domaine des études théâtrales, le courant de recherche des performance studies (Schechner, 2013) a montré la nécessité de développer une analyse du théâtre non pas seulement à partir du texte, de son interprétation et de la représentation publique, mais en étudiant la dimension de performance au cœur de cet art vivant (Pavis, 2014). Un aspect de cet art vivant reste toutefois encore peu exploré, l'activité de répétition, sans doute en raison de l'importance accordée à performance publique finale et à la réception. Les travaux insistent sur la relation des comédiens avec le public, laissant de côté le travail collectif de la création. L'importance des répétitions ne cesse cependant de grandir en raison de l'évolution contemporaine du spectacle théâtral qui privilégie le travail de plateau (Pignon, 2015). Depuis quelques années, un ensemble de recherches, les rehearsal studies (McAuley, 1998, 2012), leur consacre d'ailleurs une attention particulière. De leur côté, les recherches en linguistique interactionnelle sur le théâtre se sont surtout intéressées à la relation des actions scéniques avec le cadre des interactions ordinaires (Stucky, 1988, 1993 ; Verdier, 2019), en vue d'en montrer les similitudes avec la conversation (Stucky, 1994 ; Piazza, 1999), ou au contraire en souligner l'organisation spécifique (Schmidt, 2018). Les analyses conversationnelles étudient les modalités de transformation du texte en performance (Hazel, 2018) et la manière dont les interprètes s'appuient sur les connaissances de la conversation ordinaire, y compris les ressources multimodales (Norrthon, 2019), pour l'incarner dans le temps et l'espace (Lefebvre, 2018). Cherchant à saisir la « machinerie » théâtrale (Kantor, [1977] 2004), cet article est consacré plus spécifiquement aux consignes que les metteurs en scène adressent aux comédiens et leur 
traitement par ces derniers. L'analyse explore la nature du travail collaboratif en répétition, dans le temps et l'espace de la performance, qui vise l'appropriation par les comédiens et les comédiennes de patterns de jeu adéquats. On s'attache plus particulièrement aux consignes (Amerine and Bilmes, 1988 ; Depperman, 2018a ; Garfinkel, 2002 ; Mondada, $2009,2014)$ du metteur en scène lorsqu'elles sont destinées à inviter les comédiens à modifier leur jeu. On cherche en particulier à comprendre les raisons pour lesquelles le metteur en scène peut considérer que le jeu du comédien manifeste une "intention » inappropriée.

Cet article s'appuie sur une enquête ethnographique menée au sein d'une troupe de théâtre, incluant l'observation et l'enregistrement audiovisuel des différentes activités du collectifi. On s'est intéressé aux diverses phases de travail théâtral - lectures à la table, répétitions et performances publiques - durant lesquelles les metteurs en scène et les comédiens mettent en place une représentation publique. Un corpus d'enregistrements audiovisuels permet de documenter les ressources sémiotiques et interactionnelles qui contribuent au travail de création. Il est transcrit selon les conventions de l'analyse de conversation d'inspiration ethnométhodologique (Mondada, 2018, voir les conventions utilisées à la fin de cet article).

$\mathrm{Au}$ cours d'une répétition, les " intentions » que le metteur en scène attribue au comédien se produisent fréquemment en réaction au jeu de ce dernier et s'accompagnent d'instructions relatives aux techniques de jeu ${ }^{i i}$. Nous étudions en ce sens les répétitions de la pièce Je veux seulement que vous m'aimiez, écrite par le metteur en scène qui s'est inspiré du travail du cinéaste et dramaturge allemand R. W. Fassbinderiii. Ces répétitions suivent un travail d'écriture et d'improvisation qui s'est déroulé sur plusieurs semaines. Le texte étant désormais fixé (même si le metteur en scène est amené à le modifier au vu de ce qu'il se passe en répétition) il est déjà bien connu des comédiens. Après la phase d'improvisation où le metteur en scène a encouragé ces derniers à faire des propositions, celle qui nous intéresse ici se situe à un moment où il est amené à préciser ses attentes.

L'analyse s'appuie sur une situation de répétition particulière : la scène explorée en détail dans cet article se déroule dans une boite de nuit et implique directement deux personnages, Marlène et Jorgos. L'objectif est de comprendre le format interactionnel des consignes dans leur multimodalité et leur déploiement séquentiel. La séquence, extraite de la répétition d'une pièce écrite par le metteur en scène, présente une relative cohérence dans son déroulement. L'article, qui reprend l'organisation chronologique de la séquence, s'articule en quatre sections. : (1) alors que le comédien en jeu indique la transition vers une nouvelle scène, le metteur en scène intervient et initie une période de commentaires (section 1) ; (2) le metteur en scène intervient ensuite pour fournir une série d'évaluations et de consignes de jeu (sections 2 et 3) ; (3) la séquence se termine lorsque le metteur en scène lui-même en signale la fin en introduisant une remarque conclusive (section 4).

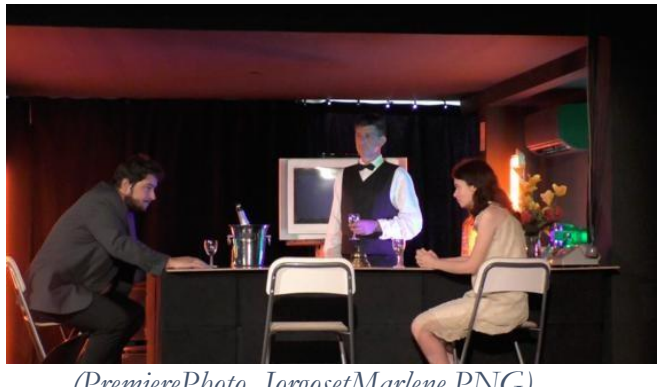

(PremierePhoto_JorgosetMarlene.PNG)

Jorgos (gauche) à Marlène (droite) : "J'aime pas la nature. » Debout derrière le comptoir, le barman essuie les verres. 
Les deux personnages, assis au bar derrière lequel se tient un barman occupé à nettoyer les verres, évoquent un possible futur ensemble. Lorsque Marlène exprime son désir de vivre dans la forêt, Jorgos exprime une très forte opposition, expliquant qu'il n'aime pas la nature, parce qu'il trouve que la nature ou la forêt " c'est pas un endroit où on peut fuir ». Les indications dans le texte écrit stipulent qu'après avoir dit sa réplique, le personnage se lève, prend la bouteille et quitte Marlène pour aller retrouver un autre personnage, Ali, marquant ainsi le passage à une autre scène.

Le texte écrit sur lequel s'appuient les comédiens est le suivant :

Jorgos en s'énervant :

J'aime pas la nature.

Je trouve que la nature... je sais pas... (plus intérieurement)

Je trouve qu'une forêt,

ou ce que tu veux

c'est pas un endroit où on pourrait fuir.

Marlène : Mais je ne veux pas fuir, Jorgos.

Jorgos : La nature c'est un endroit où on ne peut pas fuir. Tu comprends ? ${ }^{\text {iv }}$

Marlène : La nature, c'est joli, c'est sympathique.

Jorgos : Sympathique si veux.

Moi je préfère laisser ça de côté ! Merde!

Il se lève en prenant la bouteille et s'en va vers Aliv.

Les deux sections qui suivent examinent les techniques de direction du metteur en scène visant à rectifier une proposition de jeu, celles-ci émergeant juste après l'extrait que nous avons introduit précédemment.

\section{Section 1. Évaluation du jeu du comédien}

On note que les consignes présentent généralement le format séquentiel suivant : une évaluation précède généralement l'énoncé d'une consigne. C'est le cas dans le premier extrait de la séquence étudiée. Au moment où l'acteur-Jorgos énonce « la nature c'est pas un endroit où on pourrait fuir », le metteur en scène dit " allez » en claquant des doigts pour encourager les comédiens à être plus énergiques. Puis il les interrompt et produit une évaluation de la performance, en prenant tout particulièrement pour cible la manière dont le comédien s'est éloigné du comptoir, "en s'énervant " comme le précise le texte. La performance ne «marche pas » (L. 1), explique le metteur en scène, parce que le comédien a « triché » (L. 4-5).

JVSQVMA_131018_1vi

1. MS \#y a un truc qui marche pas A (.)

\#MS marche vers le comptoir où les deux acteurs sont assis-->

3. c'est que: : euh (0.3)

4. c'est juste, mais euh tu triches. (0.5)

5. C'est involontaire tu triches pas exprès j'veux dire mais $(0.8)$

Étant donné qu'il n'est pas rare pour les comédiens de s'appuyer sur des trucs qu'ils ont mis au point au cours de leurs pratiques de jeu, l'évaluation du metteur en scène, qui prend la forme d'un reproche, mérite considération : il invite le comédien à examiner ce qu'il vient de faire, en admettant que, bien qu'il l'ait fait de manière involontaire (L. 5), il a quand même «triché ». Le metteur en scène fait référence au fait que lorsqu'il s'est levé pour rejoindre Ali, il a accompli cette série d'actions tranquillement, en attrapant la bouteille et en s'éloignant d'un mouvement lent. Il faut tenir compte ici de l'arrière-plan partagé des interlocuteurs que nos observations permettent de préciser : dans ses sorties de scène, le comédien a l'habitude de ce type de comportement. Le metteur en scène fait donc allusion ici au fait que le comédien n'hésite pas à utiliser une de ses routines de sortie de scène : une démarche chaloupée et lente. Au lieu d'introduire une brusque rupture de la relation avec Marlène, sa performance indexe plutôt un retrait global de l'action dramatique, ce qui est, selon le metteur en sçène, une manière inadéquate de considérer le 
comportement de son personnage. Alors même que le metteur en scène vise un acte intentionnel de la part du comédien, ce même acte est évalué comme " involontaire » et non-délibéré (L. 5). L'interprétation d'un rôle suppose toujours en effet que le comédien choisisse parmi un éventail de possibilités d'actions. L'utilisation de sa routine de jeu habituelle l'a empêché de développer une sortie de scène qui soit adaptée à l'exécution scénique que demandait le texte.

La forme de jeu proposée aux comédiens par le metteur en scène s'appuie sur un texte décrivant des situations quotidiennes (des échanges entre amis dans une boite de nuit), et vise à transposer des interactions ordinaires sur scène (Stucky, 1993) : parler d'amour, danser, raconter un film qu'on a beaucoup aimé, se disputer, etc. La conception adéquate du jeu telle que les comédiens et le metteur en scène le conçoivent ici est celle d'un jeu « naturel », pour lequel le jeu s'inscrit dans la réalité du faire ${ }^{\text {vii }}$ (Meisner 1987 : 16). Pour le comédien, cela implique : " to think, strive, feel and act in unison with [their] role » (Stanislavski, 1948 : 14). Il s'agit donc pour l'analyste de saisir la manière dont les comédiens parviennent, de manière collaborative, à jouer à l'unisson de leur rôle, dans l'espace et le temps de la scène.

Simulant des activités ordinaires, les comédiens et les comédiennes sont alors très sensibles au fait que le public ainsi que leurs partenaires de jeu sont susceptibles d'attribuer un sens au moindre de leurs faits et gestes. Le jeu d'acteur nécessite alors un ajustement conscient entre ce qui est dit, les gestes, les actions et les déplacements sur scène. Un tel ajustement requiert un travail intensif durant les répétitions, et les metteurs en scène, chargés d'en vérifier la justesse, présentent à tout moment des formes de raisonnement qui s'appuient sur le comportement des comédiens pour en inférer leurs intentions : en ce sens, la question de l'intention dans l'action est un phénomène observable. La direction d'acteur vise à ce que les comédiens soient particulièrement attentifs à l'image qu'ils construisent lorsqu'ils sont sur scène, et c'est pourquoi les discours métapragmatiques commentant le jeu de l'acteur sur scène implique l'explicitation des inférences particulières qui émergent des actions afin de corriger de possibles interprétations erronées ou indésirables de la part du public. C'est le cas lorsque, comme dans cet extrait, le metteur en scène signale une intention non-ajustée à l'action qui semble émerger d'une proposition de jeu de l'acteur : les actions du comédien sont alors évaluées en termes de " correct (accepted) and wrong (unaccepted) inferences » (Depperman, 2018b). Le metteur en scène distingue nettement entre les intentions du comédien et les intentions attribuées par le public (Dynel, 2016) en soulignant que les actions que le comédien a l'intention d'impliquer ne coüncident pas forcément avec celles que le public saisit effectivement. Les instructions scéniques opèrent ainsi ici à la fois au niveau des interactions entre les personnages, et à celui de la réception du public, susceptible de tirer des inférences.

\section{Section 2. Suite de l'évaluation du jeu du comédien et première consigne}

Poursuivant ses commentaires sur la proposition de jeu de l'acteur qu'il a initiés dans le premier extrait précédemment étudié, le metteur en scène évalue cette fois positivement ce qu'il vient de faire affirmant qu'il l'a fait intentionnellement (et non par hasard ou inconsciemment) : « ton intention est juste » (L. 5).

JVSQVMA_131018_5_13

5. MS *ton intention est juste, (.)*\#

* Ies deux avant-bras projetés en avant*

6. \#*mais tu laisses pas ton corps jouer $\backslash *($.

\#debout à côté du comédien assis ------->>

*mouvements des bras et du corps* 


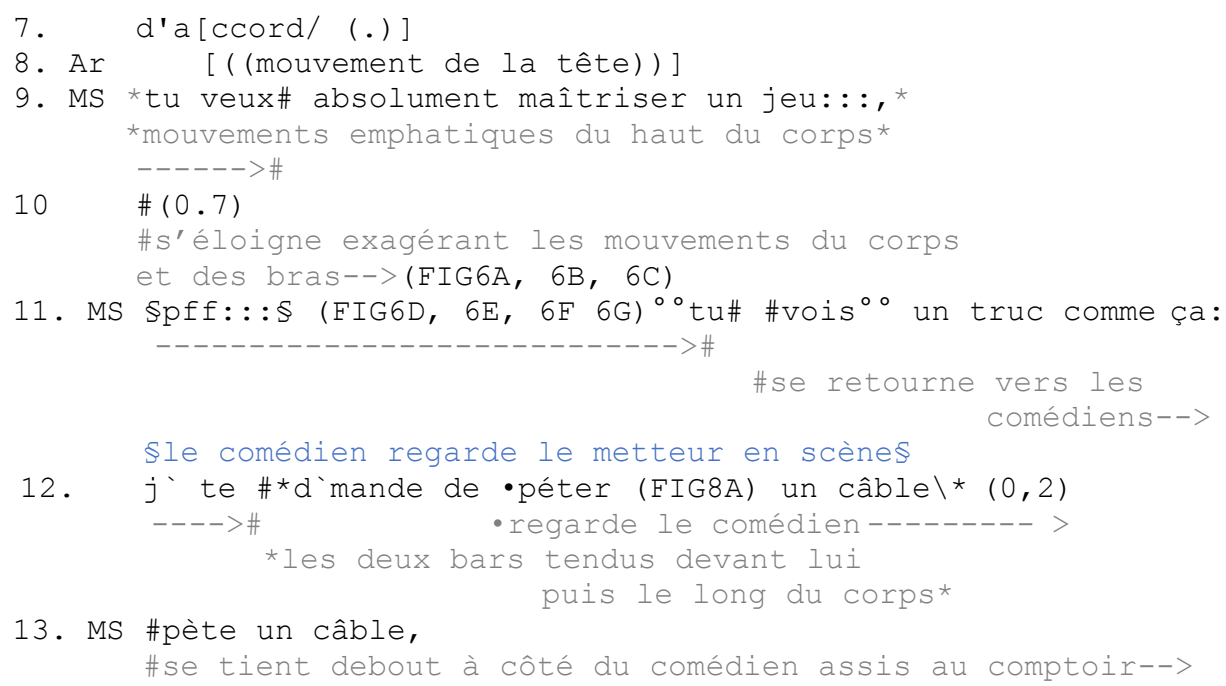

Cette évaluation positive est immédiatement suivie d'une évaluation négative concernant le corps de l'acteur (L. 6 « mais tu laisses pas ton corps jouer ») qui souligne un décalage entre l'intention prêtée au comédien - s'énerver - et sa performance - un mouvement lent pour s'éloigner du comptoir. Cette évaluation se conforme à l'idée de sens commun selon laquelle les intentions participent des motifs d'agir et président aux actions de la vie ordinaire (Depperman, 2012 : 746). Il n'est donc pas étonnant de trouver de la part du metteur en scène une formulation de la performance en termes psychologiques : pour améliorer la précision et l'habileté du comédien, il est nécessaire que l'action soit exécutée de manière consciente et délibérée, même si par ailleurs les effets de celle-ci restent non intentionnels (voir ce qui est dit avant l'évaluation, ligne 4).

Le metteur en scène, cherchant à vérifier l'accord du comédien sur l'évaluation de sa performance, formule une requête, d'accord? (L. 7), qui projette une réponse affirmative et s'assure de l'alignement de son interlocuteur. L'acteur fournit une deuxième partie de paire affiliative à la demande de ratification, minimalement, d'un léger mouvement de tête (L. 8). Le metteur en scène poursuit alors son évaluation sous la forme d'un « assessment description » (Goodwin and Goodwin, 1992, 2004), " tu veux absolument maîtriser un jeu » (L. 9), qui dévoile l'agenda supposément caché du comédien : la maîtrise entière de son jeu. Cette évaluation est négative - les mouvements emphatiques (un haussement des épaules) du metteur en scène ainsi qu'une intonation traînante signalent le désalignement du metteur en scène (Goodwin and Goodwin, 1987 : 3 ; Couper-Kuhlen, 1996). Cette évaluation est aussitôt suivie d'une manière exagérée de marcher (L. 10), passant d'une description de l'agir du comédien à une imitation qui exagère certaines de ses actions lorsque ce dernier s'est éloigné du comptoir. 


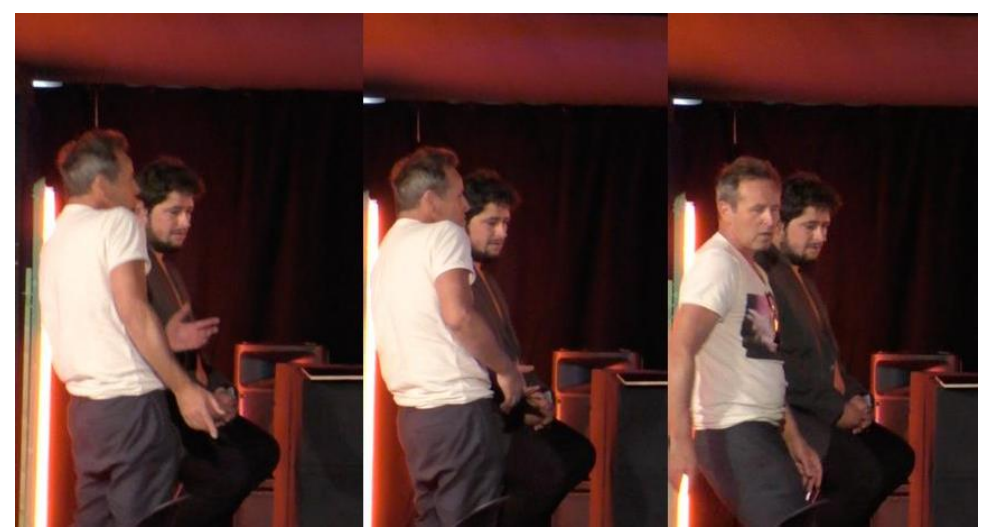

(FIG 6A, FIG 6B \& FIG 6C) Le metteur en scène imite la performance du comédien (début).

L'imitation ne suppose pas nécessairement de reprendre les expressions verbatim ou d'exécuter exactement les mêmes mouvements de la personne que l'on imite ou parodie (Rossen-Knill Henry, 1997 : 727). Le metteur en scène rend saillant des éléments de la performance de l'acteur qu'il est censé reprendre, en marchant avec des mouvements de hanche très accentués, tout en ajoutant des éléments, comme l'onomatopée «pff » (L. 11) le comédien n'ayant pas émis de son particulier lors de sa performance. Le metteur en scène contribue ainsi à présentifier l'attitude émotionnelle du personnage que lui semblait devoir communiquer la performance du comédien - elle est ici explicitement exprimée principalement par les mouvements du corps. Les segments intonatifs marquant une voix traînante qui caractérisent la prononciation de son évaluation ( "u veux absolument maîtriser un jeu », L. 5), s'ils contribuent à accentuer la dimension très négative de celle-ci, commentent en même temps l'attitude du personnage.

Arrêtons-nous un instant sur la voix traînante qu'adopte le metteur en scène. Elle a pour but de parodier son propre jeu, i.e. une exécution scénique trop lente. La dimension négative de l'évaluation du jeu de l'acteur est produite au moyen d'une prononciation traînante, qui en conséquence est liée, non, comme on l'attendrait, à ce qui est dit, mais aux mouvements du corps qui sont réalisés de façon concomitante.

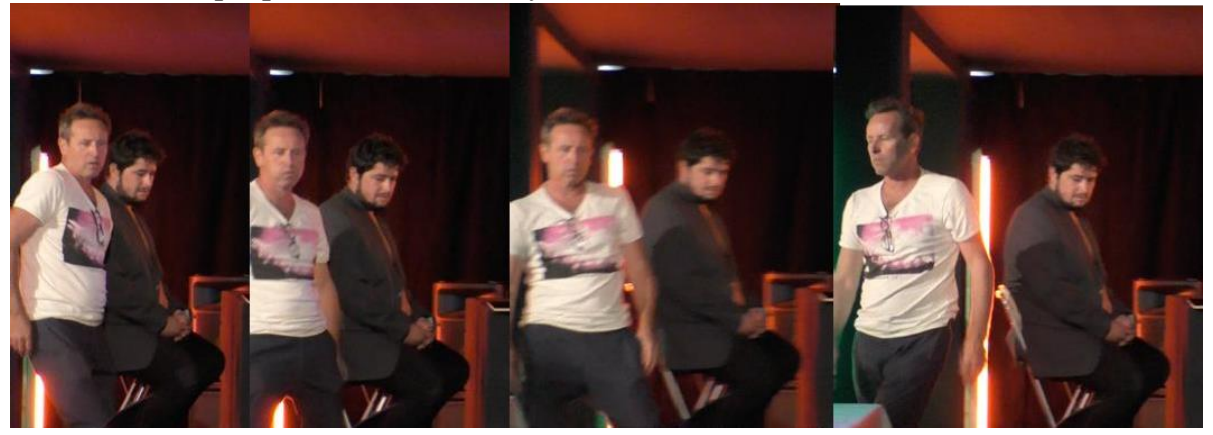

(FIG 6D, FIG 6E, FIG6F \& FIG 6G)

Le metteur en scène imite la performance du comédien (suite et fin).

Il produit une action en s'appuyant sur ce qui a été fait par le comédien en adoptant la perspective supposée de celui-ci, et dans le même temps il transforme le comportement original dans un nouveau contexte en ajoutant sa propre perspective critique sur ce qu'il s'est passé : de ce point de vue, il agit à la manière d'un parodiste qui prend ses distances par rapport à l'objet de la parodie (Rossen-Knill et Henry, 1997 : 728). Il adopte une certaine position par rapport à ce qu'il représente (Selting, 2010), à savoir que les mouvements du corps du comédien étaient lents et paresseux, tout en donnant 
simultanément son avis sur la manière dont l'acteur a voulu dépeindre la colère. Il transmet ainsi, de manière multimodale, à la fois la nature de la figure que la performance a créée, celle d'un personnage détaché et désabusé, et la critique du jeu de scène, un jeu maniéré et ostentatoire. On remarque que la désaffiliation qu'il présente par rapport à ce qu'il imite (Goffman, 1974 ; Couper-Kuhlen, 1996 ; Mondada, 2009) est clairement perçue par le comédien (L. 11).

Lorsqu'il s'approche du comédien en le regardant (ligne 12), il formule très clairement ses attentes : « je te demande de péter un câble ». Dans un tel contexte où l'auteur de la demande a toute autorité sur la situation, " demander » a pour fonction d'ordonner au comédien à agir d'une certaine manière. Le metteur en scène répète ensuite la même recommandation, " pète un câble » (ligne 13), maintenant sous la forme d'une directive. Lorsqu'il ordonne au comédien d'accomplir cette action, très générale, passant d'une évaluation à la formulation d'une instruction, il s'appuie sur les connaissances que l'acteur et lui partagent nécessairement, i.e. la nécessité, pour les participants, pour incarner le script écrit, de s'appuyer sur leurs connaissances des pratiques de la conversation quotidienne (Norrthon, 2019) en fonction des conventions qui permettent d'interagir dans le monde ordinaire (Lyon, $1982: 82$ ).

Néanmoins, l'action de « péter un câble » compte un large éventail d'actions possibles, et elle n'est pas si simple à mettre en œuvre dans le temps et l'espace de la scène. Lorsque le metteur en scène exprime l'évaluation de ce qui a été proposé par le comédien, il le fait de manière très précise, en rejouant ce qui a été fait tout en ajoutant sa propre évaluation au travers différentes modalités, gestuelles, prosodiques et vocales. Néanmoins, le fait qu'il accentue certains traits sans en mentionner un en particulier qui serait particulièrement problématique, n'est pas sans conséquence. Ainsi, quelques secondes après, le comédien émet une demande de clarification : « c'est dans la marche? » (L. 22), suggérant qu'il n'est pas tout à fait certain de l'élément de jeu auquel la consigne réfère en particulier (L. 22) : JVSQVMA_131018_16_24

16. MES quand tu pètes un câble tu sais pas c’que ton corps va - faire,

17. oui ou non

18. et là tu sais • très bien c'que tu vas fairel

19 *jouer ton doigt: : *

20. tu peux *arrêter *ton \#intention et Smarcher comme ça \\# *les deux mains sur l'estomac *

\#s'éloigne d'un pas nonchalant\#

\$le comédien regarde

21. \#tu vois, (.) le MES ----->

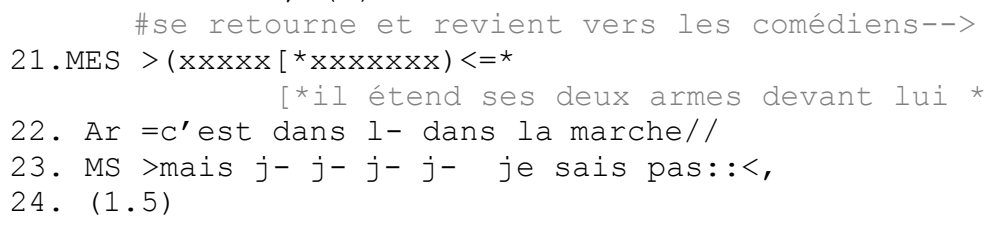

Le metteur en scène vient d'expliciter son évaluation critique précédente qui portait sur la maitrise du jeu de l'acteur (« tu laisses pas ton corps jouer », (L. 6) suivi de « tu veux absolument maitriser un jeu » (L9)). Après avoir expliqué que la colère implique de ne pas chercher à maitriser ou à anticiper les mouvements du corps (L16), il reprend l'imitation de la performance, sur le même modèle que celui décrit précédemment : il produit l'action de 
pointer du doigt (L. 19) et marcher (L. 20) en adoptant la perspective de l'acteur, tout en modifiant les actions d'origine en indiquant sa propre perspective. La dimension critique est formulée par les gestes (le doigt pointé vers la comédienne que l'acteur a utilisé pour exprimer sa colère) et les mouvements du corps (un pas nonchalant) : pointer un doigt lorsqu'on est en colère est un signe démonstratif très stéréotypé qui rompt le contrat « naturaliste »; le pas nonchalant lorsqu'on est censé exprimer un mouvement de colère instaure quant à lui une distance entre les émotions du personnage et le comportement qui lui est associé. Cette critique est comprise par le comédien qui répond positivement à la demande de ratification émise par le metteur en scène (L. 21).

Pour autant, à ce stade, l'évaluation, et la consigne qui lui est liée, échouent. Le comédien ne parvient pas à élaborer à partir de ce qu'a proposé le metteur en scène. Ayant prêté attention aux instructions qui lui étaient proposées, il a perçu que sa façon de bouger était en cause, mais il n'a pas exactement compris la nature du problème. Sa requête " c'est dans la marche? " (L. 22) - indique qu'il n'a pas saisi que le metteur en scène souhaitait une performance à tous les niveaux, corps, voix et mouvements. Alors que la demande du comédien pointe vers le détail de la marche, la performance du metteur en scène a au contraire souligné que l'expression de la colère doit émerger de tout le corps, et qu'un changement de comportement au niveau de la marche serait par conséquence à la fois insuffisant et inadéquat.

\section{Section 3. Deuxième consigne}

Suite à la demande de clarification du comédien (L. 22), le metteur en scène l'invite alors à rejouer la scène avec beaucoup plus d'intensité (quelques lignes sont omises ici, lorsque le metteur en scène joue la scène avec le comédien en prenant le rôle féminin). Pour initier de nouveau la scène, il demande à la comédienne de dire sa réplique (L. 45), " c'est joli la nature c'est sympathique » (L. 46), à l'origine de l'explosion de colère de Jorgos.

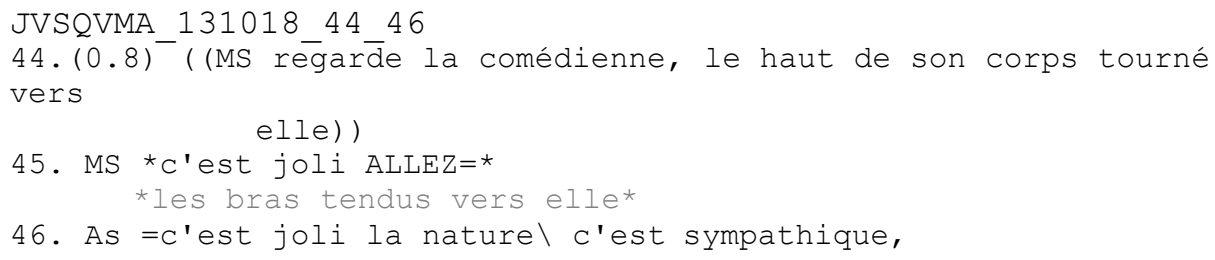

Le comédien enchaîne sa propre réplique (L. 47) après celle de sa partenaire de jeu (L. 46).

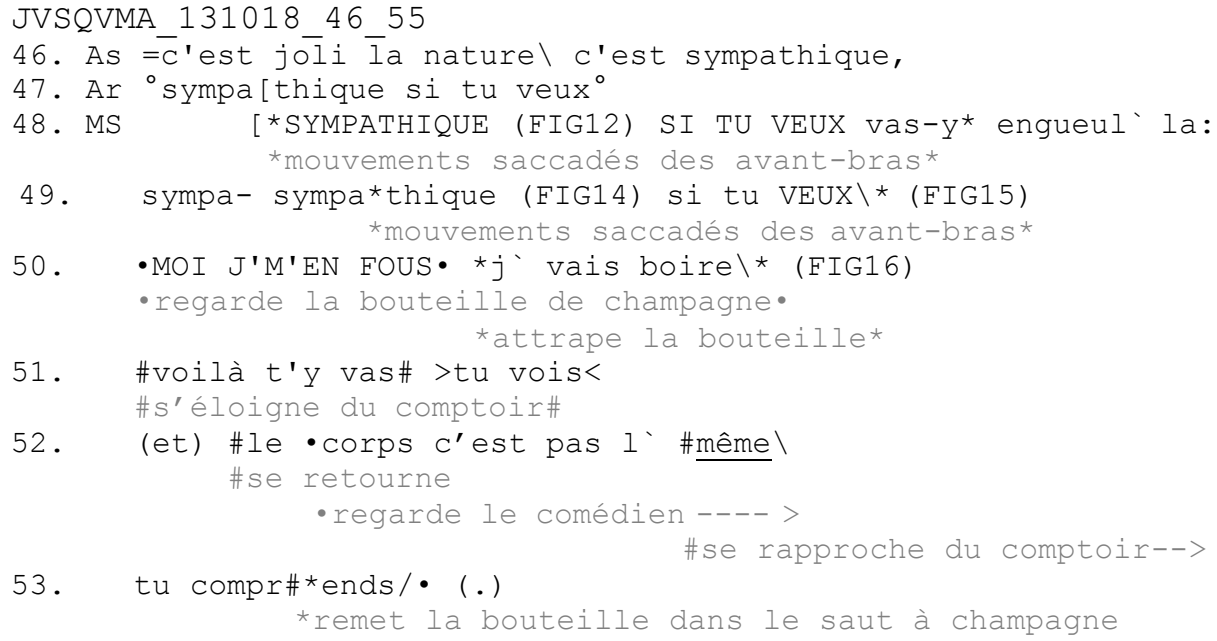




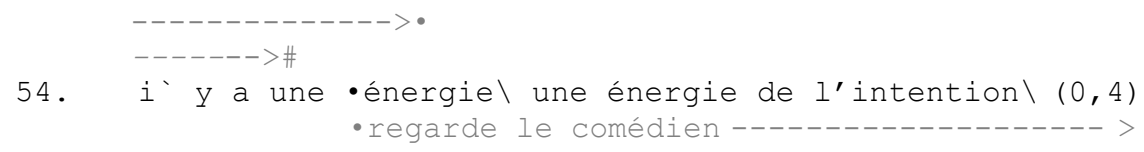

Que la performance de la comédienne ne soit pas satisfaisante importe peu ici car elle vient en appui du comédien, sa remarque sur la nature étant le déclencheur de la colère du personnage. A ce stade, ce sont les modalités scéniques de l'expression de cette colère qui constituent le sujet des consignes du metteur en scène.

Alors que le comédien prononce sa réplique à voix basse, "sympathique si tu veux » (L. 48), en restant immobile, le metteur en scène produit la même réplique, en chevauchement (L. 48). Cette prise de parole en chevauchement ne provoque pas de rupture au niveau du cadre de participation créé par les répliques des deux comédiens, le metteur en scène projetant une simple reformulation de l'action du comédien. La manière dont il recycle la réplique de l'acteur, avec un changement dramatique dans l'intensité de la voix et des mouvements saccadés des bras, tranche par rapport à l'immobilité et au ton bas du comédien.

En revanche, la consigne qui suit, sous la forme d'une directive, « vas-y engueule-la » (L. 48), opère immédiatement une rupture du cadre. Ce segment recadre la première partie de l'énoncé («sympathique si tu veux », L. 48) comme étant également une instruction, en indiquant dans le même temps que le comédien n'a toujours pas saisi le sens de ses consignes (Depperman, 2012). Le metteur en scène enchaîne en réitérant la même réplique (" sympathique si tu veux », L. 49), ajoutant, « moi j'm'en fous, j'vais boire » (L. 50), prononcée d'une voix forte, qui est une interprétation libre du texte de la pièce (dans lequel il est écrit : « Moi je préfère laisser ça de côté ! Merde ») viii.

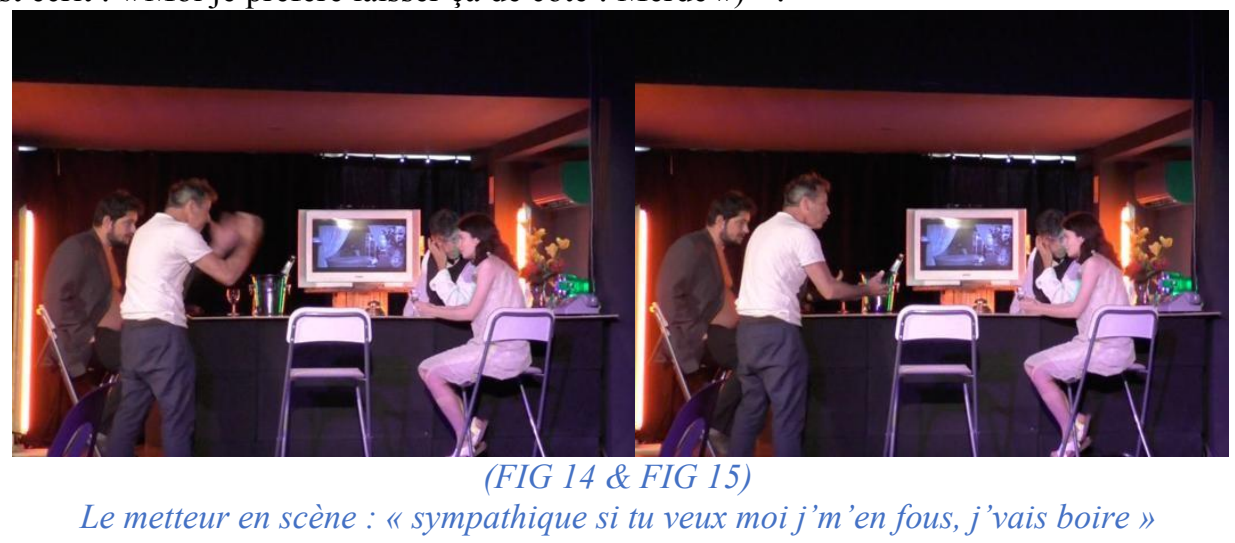

Alors qu'il prononce ces mots (L. 50), il regarde la bouteille de champagne sur le comptoir, la saisit et s'éloigne. Ses gestes sont brusques et rapides. 


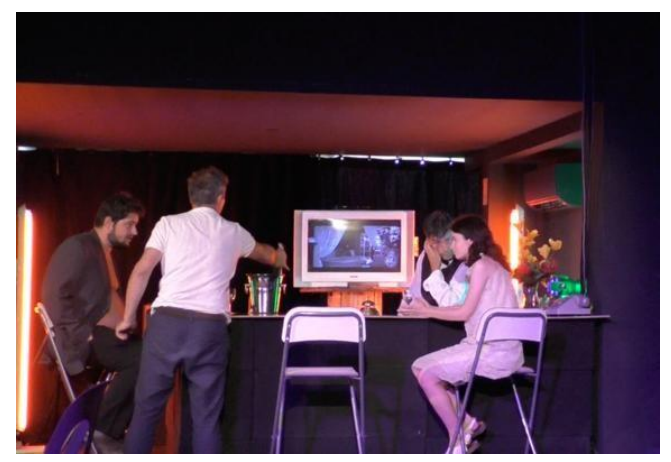

(FIG 16) Le metteur en scène attrape la bouteille sur le comptoir.

Les mouvements du corps et la vitesse d'action enrichissent l'énoncé initial de la consigne qui était « vas-y engueule-la » (L. 48). Le metteur en scène produit une nouvelle forme de jeu qui contraste avec le jeu du comédien, sa performance présentant des choix d'interprétation très clairs : une voix forte, une brusquerie dans les mouvements, la rapidité des actions, qui formulent l'action globale - se mettre en colère contre quelqu'un - au travers de l'action globale secondaire qu'il vient de performer. L'acteur est ainsi invité à inférer que ce qu'il a fait précédemment n'était pas adéquat : le metteur en scène invite le comédien à jouer la séparation brusque avec le personnage féminin, en engageant toute l'énergie du corps et de la voix.

Commentant ensuite ce qui vient de se passer (ligne 51), le metteur en scène explique que ce qu'il a fait doit être compris comme une consigne liée d'abord au corps, « et le corps c'est pas le même » (L. 52). Après avoir cherché la confirmation que cela a bien été compris, d'abord par le regard, puis explicitement (ligne 53), il produit une reformulation de la déclaration précédente (ligne 52) à un niveau plus général, celui de l'intention : « il y a une énergie de l'intention » (ligne 54). Cela constitue une conclusion, ou un résumé, de la question en jeu depuis le début de cette séquence, à savoir que l'action que suppose le texte (l'intention) et l'action réalisée en scène nécessitent toute l'énergie du comédien pour être congruentes et ajustées entre elles.

\section{Section 4. Évaluation finale et conclusive}

Le metteur en scène produit alors une suite d'énoncés généralisant sur les comédiens : «c'est un problème typique des acteurs français, c'est des petits bâtons qui parlent, qui ont pas de corps » (L. 65-67).

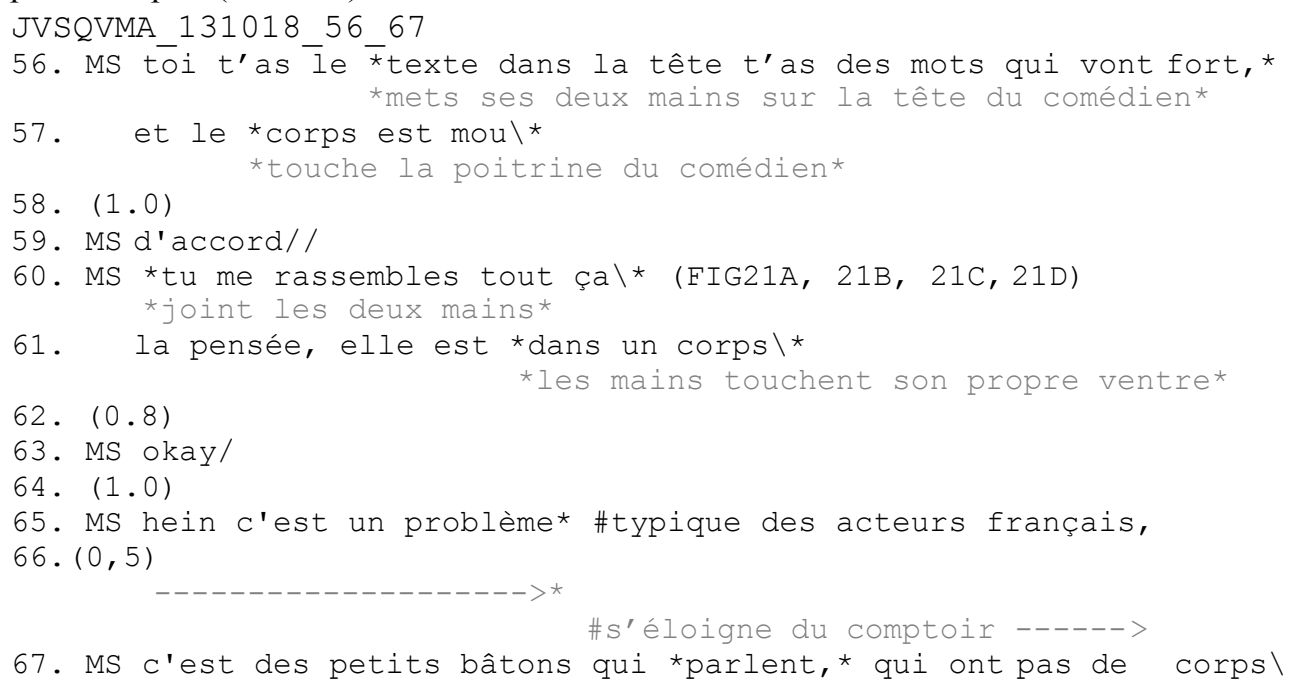




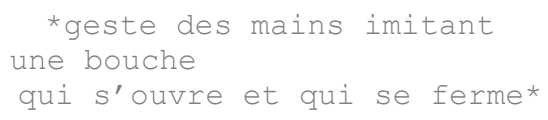

Le problème de l'intention, tel qu'il a été introduit au départ - « ton intention est juste mais tu laisses pas ton corps jouer » (L. 5-6) - est maintenant repris dans l'opposition entre le corps et l'esprit : " toi t'as le texte dans la tete et les mots qui vont fort et le corps est mou » (L. 56-57).

Que l'interprétation ait tendance à passer d'abord par le texte (on pense effectivement à l'importance que prend aujourd'hui la « lecture à la table ») puis à être incarnée sur scène, est à mettre au compte d'une conception de la performance théâtrale largement répandue, " typique des acteurs français ». Selon le metteur en scène, la division corps-esprit empêche les interprètes d'expérimenter une performance globale (ce que la gestalt montrait en L. 50-51). Les mains entrelacées sur le ventre du metteur en scène (L. 61-62) résument gestuellement cette interconnexion nécessaire du texte et du corps pour une interprétation réussie : « la pensée elle est dans un corps » (L. 61). La non congruence entre l'intention du texte et les actions du comédien renverrait à une conception fausse qu'ont en général les acteurs français, d'une séparation de la pensée (assimilée aux intentions supposées être à l'origine des actions) et du corps : cette opposition explique la manière dont le metteur en scène présente ses deux séries d'instructions, sur lesquelles nous revenons en conclusion.

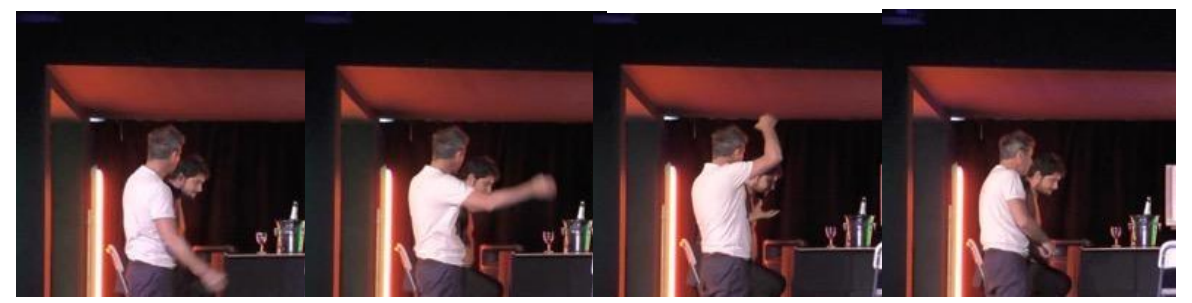

(FIG 21A, 21B, 21C\&21D)

Les deux mains se rejoignent, figurant ainsi le rassemblement du corps et de la pensée.

\section{Discussion et conclusion}

Cet article a analysé les caractéristiques interactionnelles de deux techniques de consignes de jeu qu'un metteur en scène est amené à développer lors d'une répétition, et qui renvoient toutes deux à un problème de désajustement entre l'action nécessitée par le texte et l'action réalisée sur scène. La première technique s'appuie directement sur la performance du comédien, et consiste à produire une évaluation négative, tant sur le plan grammatical (marqueurs de négation) que sur le plan multimodal (imitation exagérée), sélectionnant certains traits, en laissant à charge pour l'acteur de modifier son jeu. Dans ce format, l'évaluation prédomine et la consigne est tacite : le comédien sait maintenant ce qu'il ne doit pas faire, mais il lui reste à trouver une exécution scénique adéquate. La deuxième technique indique quant à elle qu'il y a un problème et propose une solution sous la forme d'un pattern de jeu que le comédien est invité à reprendre. Dans ce format, la consigne se présente sous une forme positive (une proposition de jeu), mais elle a pour conséquence de réduire la marge d'interprétation du comédien. Les deux techniques supposent, soit de souligner certains traits, problématiques, de la performance initiale (première technique), soit de présenter de façon incarnée quelques-unes des caractéristiques d'une interprétation adéquate (seconde technique). Une proposition d'exécution scénique émerge ainsi progressivement dans l'ici et maintenant du plateau.

La première technique du metteur en scène implique la reprise globale du jeu de l'acteur. Le risque est alors que le comédien ne soit pas en mesure de déterminer ce qu'il doit modifier dans son jeu (cf. la requête de clarification L. 22). L'avantage est qu'elle permet de laisser de la marge d'interprétation au comédien. Après que celui-ci ait demandé ce qui devait être modifié, le metteur en scène reformule alors sa consigne, cette fois en 
proposant sa propre interprétation. Cette seconde technique, en indiquant ce qu'il faut faire, est relativement autoritaire et contraignante. Les metteurs en scène, généralement, préfèrent réserver une part d'indétermination dans leur proposition de jeu pour laisser le maximum d'initiative aux comédiens. Pour cette raison, le metteur en scène reformule le texte, une technique qui permet d'atténuer la directivité de sa consigne. Il signale par ailleurs ainsi que le problème ne concerne pas tant le contenu que la manière dont le texte est énoncé - le pattern prosodique et rythmique - et incarné - les mouvements du corps, etc.

L'extrait de cette répétition nous permet d'observer les modes d'articulation entre la perception par le metteur en scène des actions du comédien et les attributions d'intentionnalité dérivées directement de l'observation de la performance du comédien. Les techniques du metteur en scène impliquent d'incarner, dans le temps et l'espace scénique, l'intention qu'il suppose à l'acteur et les actions que ce dernier devrait mettre en œuvre pour être en adéquation avec les actions prescrites par le texte. Les répétitions permettent ainsi d'étudier les procédures d'ajustement entre les actions à accomplir telles qu'elles sont évoquées dans le texte et comprises par l'interprète, avec les actions effectivement performées sur scène. Pour une performance réussie, les instructions comprises dans le texte et l'action à réaliser doivent coïncider : toutefois, les indexer explicitement ou implicitement à travers des dispositifs linguistiques ne suffit pas, le corps doit également être pleinement impliqué.

Les actions que les comédiens doivent déployer sur scène ne sont pas données d'emblée, mais requièrent un travail d'expérimentation sous l'œil du metteur en scène et des autres comédiens. Pendant les répétitions, il est très difficile pour le comédien de juger du résultat et de proposer des modifications in situ de son jeu. C'est le rôle du metteur en scène de l'aider à y parvenir : d'où l'importance de son regard, de ses observations et de sa capacité à proposer des solutions qui implique autant de proscriptions que de prescriptions. Dans la séquence étudiée, le metteur en scène demande au comédien de changer radicalement son jeu. L'accomplissement d'actions scéniques nécessite ainsi tout un travail conjoint. Le travail du comédien et du metteur en scène est d'arriver, par de multiples répétitions, à trouver l'action appropriée sur scène (Hazel 2018) - déclarer son amour, donner une gifle, courir aller mettre un disque dans le jukebox, jeter un bouquet de fleurs par terre avec colère, raconter la scène d'un film qu'on a aimé, etc. - tout en donnant l'impression qu'elle est produite de manière spontanée et imprévisible. Autrement dit, « le drame sur scène ne doit pas « se passer » mais «devenir », se développer sous les yeux du spectateur »(Kantor, [1977] 2004 : 34-35). Les actions sur scène étant basées sur un ensemble de conventions, le metteur en scène doit introduire des instructions spécifiques relatives aux attentes d'arrière-plan pour que ces conventions soient accomplies de manière adéquate. L'une des conventions de l'œuvre dans cette pièce est celle d'un jeu « naturel », qui suppose une relation entre la manière de se conduire sur scène et l'action qui se déploie sur scène. Conséquence : si, dans la vie ordinaire, les participants s'appuient sur les ressources de la situation pour interagir, sur scène en revanche, il faut tout un travail supplémentaire (métapragmatique) pour parvenir à faire oublier que les comédiens connaissent à l'avance l'enchaînement des actions des personnages et faire en sorte que ces actions se déroulent de façon spontanée et imprévisible.

\section{Références}

Amerine R., Bilmes, J. (1988). Following Instructions. Human Studies, 11, 327-339. 
Couper-Kuhlen, E. (1996). The prosody of repetition: On quoting and mimicry. In: Couper-Kuhlen, E. (Ed.), Prosody in Conversation: Interactional Studies. Cambridge: Cambridge University Press, 366-405.

Depperman, A. (2012). How does 'cognition' matter to the analysis of talk-ininteraction?. Language Sciences 34, 746-767.

Depperman, A. (2018a). Instruction practices in German driving lessons: Differential uses of declaratives and imperatives. International Journal of Applied Linguistics 28 (2), 265-282

Depperman, A. (2018b.) Inferential Practices in Social Interaction: A ConversationAnalytic Account. Open Linguistics 4, 35-55.

Dynel, M. (2016). With or without intentions: Accountability and (un)intentional humour in film talk. Journal of Pragmatics 95, 67-78.

Garfinkel, H. (2002). Ethnomethodology's Program. Oxford : Rowman \& Littlefield.

Garner, S. B. (2018). Movement, Attention, and Intentionality. Kinesthetic

Spectatorship in the Theatre: Phenomenology, Cognition, Movement, 09-144.

Goffman, E. (1974). Frame analysis: An essay on the organization of experience, Cambridge : Harvard University Press.

Goodwin, C., Goodwin, M. H. (1987). Concurrent Operations on Talk: Notes on the Interactive Organization of Assessments. IPRA Papers in Pragmatics 1(1), 1-54.

Goodwin, M. H., Goodwin, C. (1992). Assessments and the Construction of Context. In: Duranti A., Goodwin C., (Eds). Rethinking Context: Language as an Interactive Phenomenon, Cambridge : Cambridge University Press, 147-90.

Goodwin, M. H., Goodwin, C. (2004). Participation. In: A. Duranti (Ed.), A Companion to Linguistic Anthropology, Oxford : Basil Blackwell, 222-244.

Hazel, S. (2018). Discovering Interactional Authenticity: Tracking Theatre Practitioners Across Rehearsals. In: Pekarek Doehler S., Wagner J. and González-Martínez E. (Eds.), Longitudinal Studies on the Organization of Social Interaction, London : Palgrave Macmillan, 255-283.

Jolivet Pignon, R. (2015). La représentation rhapsodique - Quand la scène invente le texte, L'Entretemps, coll. « Champs théâtral ».

Kantor, T. ([1977] 2004). Le théâtre de la mort. Textes réunis et présentés par Denis Bablet. Paris : L'Age d'Homme.

Lefebvre, A. (2018). Reading and embodying the script during the theatrical rehearsal. Language and Dialogue 8 (2), 261-288. 89.

Lyon, E. (1982). Stages of Theatrical Rehearsal. Journal of Popular Culture 16 (2), 75 -

McAuley, G. (1998). Towards an Ethnography of Rehearsal. New Theatre Quaterly 14 (53), 75-84.

McAuley, G. (2012). Not magic but work. An ethnographic account of a rehearsal process. Manchester : Manchester University Press.

Meisner, S. (1987). On Acting. New York : Random House.

Mondada, L. (2009). The Embodied and Negotiated Production of Assessments in Instructed Actions. Research on Language and Social Interaction 42 (4), 329-361.

Mondada, L. (2014). Instructions in the operating room: How the surgeon directs their assistant's hands. Discourse Studies 16 (2), 131-161.

Mondada, L. (2018). Multiple Temporalities of Language and Body in Interaction: Challenges for Transcribing Multimodality. Research on Language and Social Interaction $51(1), 85-106$.

Norrthon, S. (2019). To stage an overlap - The longitudinal, collaborative and embodied process of staging eight lines in a professional theatre rehearsal process. Journal of Pragmatics 142, 171-184. 
Pavis, P. (2014). Dictionnaire de la performance et du théâtre contemporain. Paris : Armand Colin, coll. « Dictionnaire ».

Piazza, R. (1999). Dramatic discourse approached from a conversational analysis perspective: Catherine Hayes's Skirmishes and other contemporary plays. Journal of Pragmatics 31, 1001-1023.

Proust, S. (2004). Le corps du metteur en scène. Demeter 3, 1-11.

Reason, M. (2015). Participation: Researching the 'active' theatre audience.

Participations - Journal of Audience \& Reception Studies 12 (1), 271-280.

Rossen-Knill, D., Henry, R. (1997). The pragmatic of verbal parody. Journal of Pragmatics 27 (6), 719-752.

Schmidt, A. (2018). Projections and preparations within theatrical rehearsals. In: Depperman A., Streeck J. (Eds.), Time in Embodied Interaction: Synchronicity and sequentiality of multimodal resources, Amsterdam, Philadelphia : John Benjamins, 231260.

Selting, M. (2010). Affectivity in Conversational Storytelling: An Analysis of Displays of Anger or Indignation in Complaint Stories. Pragmatics 20, 229-277.

Simmel, G. (2001). La philosophie du comédien, Paris : Circé.

Stanislavski, C. (2003 [1936]). An actor prepares. New York \& London: Taylor and Francis Routledge.

Stucky, N. (1988). Unnatural acts: Performing natural conversation. Literature in Performance 8 (2), 28-39.

Stucky, N., (1993). Toward an aesthetics of natural performance. Text and Performance Quarterly 13 (2), 168-180.

Stucky, N. (1994). Interactional silence: Pauses in dramatic performance. Journal of Pragmatics 21, 171-190.

Verdier, M. (2019). La contextualisation : un problème étique ? CORELA, en ligne : https://journals.openedition.org/corela/7613.

\section{Conventions de transcription (Mondada 2018)}

[ début d'un chevauchement entre locuteurs

$=\quad$ enchaînement rapide entre deux tours de parole

$:$ allongement syllabique

intonation soutenue

$\backslash$ OU $\backslash \quad$ chute intonative ; chute intonative forte

/ OU // montée intonative ; montée intonative forte

souligné emphase

CAPITALE volume fort de la voix

${ }^{\circ}$ mot $^{\circ} \quad$ mot ou fragment de phrase prononcé d'un ton plus bas

$>\quad$ accélération

mot- troncation d'un mot

(xxx) mots inaudibles

(.) micro-pause

(0.6) pause en secondes

\#\# délimite les descriptions des mouvements du metteur en scène (MS)

* $\mathrm{xxx}^{*}$ délimite les descriptions des gestes du metteur en scène (MS)

•- délimite les descriptions des mouvements du regard du metteur en scène

$\S$

(Ar)

--> $\quad$ continuation de l'action décrite

FIG photo correspondant à la transcription 
${ }^{\mathrm{i}}$ Nous remercions chaleureusement les acteurs et les administrateurs de la compagnie La Bulle Bleue de Montpellier, ainsi que les artistes en résidence Bruno Geslin, Evelyne Didi and Jacques Allaire, pour la confiance qu'ils nous ont accordée.

${ }^{\text {ii }}$ La question de l'intentionnalité dans le jeu de l'acteur est en effet cruciale pour les professionnels et il serait fastidieux d'énumérer les nombreux écrits des praticiens du théâtre en lien avec cette question dans le cadre de cet article. Signalons une étude récente sur cette question du point de vue de la réception du public (Garner, 2018), une façon pour ces chercheurs de contrebalancer la tendance à surévaluer la dimension intentionnelle du côté artistique, au profit de la réception (Reason, 2015). Une approche interactionnelle a l'intérêt de replacer la question de l'intentionnalité dans le travail théâtral des metteurs en scène et des acteurs comme une pratique située et incarnée.

iii Je veux seulement que vous m'aimiez, Jacques Allaire, 2017, Création novembre 2017 au Chai de La Bulle Bleue (Montpellier).

${ }^{\text {iv }}$ En gras dans le texte original.

${ }^{\mathrm{v}}$ Texte extrait du manuscrit inédit de la pièce écrite et mise en scène par Jacques Allaire, 2017.

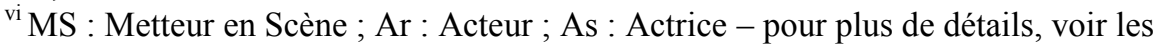
conventions de transcription en fin d'article.

vii Traduction libre de la citation originale : « acting is the reality of doing » (Meisner $1987: 16$ ).

viii Les metteurs en scène changent généralement le texte lorsqu'ils émettent des consignes, optant pour une reformulation parfois simplifiée des répliques originales. 